\title{
Emojis in Indonesian Intergenerational Family WhatsApp Group
}

\author{
Ihsan Nur Iman Faris ${ }^{1, *}$, Dian Budiarti ${ }^{2}$, Andrian Permadi ${ }^{1}$ \\ ${ }^{1}$ Universitas Pendidikan Indonesia \\ ${ }^{2}$ UIN Sunan Gunung Djati Bandung \\ ${ }^{*}$ Corresponding author. Email: ihsannif@gmail.com
}

\begin{abstract}
This study aims to investigate the use of emojis in an Indonesian Intergenerational family WhatsApp group to reveal the types and functions of emojis used in the setting where members from various gender and age groups communicate online. The participants were 26 male and 16 female WhatsApp users aged 21 to 66 years old in the group. The data were collected from five months of interaction in the WhatsApp group. The findings showed that both men and women from various age groups used the following emojis, namely 'folded hands', 'beaming face with smiling eyes', 'palms up together', 'thumbs up', and 'face with hand over mouth', in the WhatsApp group communication. In terms of function, both men and women use emojis for 1) attitude/emotion enhancer, 2) attitude/emotion signal, 3) backchanneling, 4) humour, and 5) illocutionary force. The findings contribute to research on the use of emojis relatively scarce in Indonesian contexts. The findings also suggest how to communicate using emojis in forums with members from various ages and genders.
\end{abstract}

Keywords: Age, emojis, gender, WhatsApp group

\section{INTRODUCTION}

Emojis have been used frequently in computermediated communication (CMC) to fill the absence of verbal cues and emotion signals. The use of emojis is positively viewed since it increases responsiveness, playfulness, and closeness in communication (Coyle \& Carmichael, 2019; Tang \& Hew, 2019; Prada et al., 2018; Riordan, 2017). Although ITC (information, technology, and communication) companies, such as Google and Apple, designed emojis to be used broadly and universally, the use may vary among users. People from different cultures, ages, and gender group can have a different interpretation of emojis since the pictorial words are subject to multi-interpretation (Graham, 2019). Different contexts where emojis are used can also create different meanings and interpretations. Cheng (2017) for instance, found that men tend to use more emojis in group interaction but use fewer emojis in personal communication, especially when discussing sports. Further report of how emojis have distinctive function in computer-mediated communication (CMC) is research conducted by Sampietro (2016) stating that emojis function as replacement of punctuation marks put mostly at the end of sentences.
Emoji is derived from the Japanese characters (絵, "picture") + moji (文字, 'character'). It is not taken from English word emotion (Skiba, 2016). Crystal (2001, in Alshenqeeti, 2016) suggests that the emergence of emojis' use in communication is mainly due to the nature of language responsive to changes in social, culture, needs, and tools of communication. Although CMC is a relatively new phenomenon, the use of emojis is seen as non-innovative as it tends to be highly correlated with the use of pictorial messages such as Egyptian Hieroglyphs by the past society. Responding to this point, some experts argue that the use of emojis is a form of language devolution as it indicates the return to the pre-writing era considered to be less intellectual (McIntyre, 2016).

Other experts (see for example Gülşen, 2016; Kavanagh, 2016) disagree with the notion that the use of emoji is a form of language devolution. They say that even pictorial messages are intricately complex and indicate an intellectual community of language users. They also consider the use of emojis presently is a form of language users' creativity as they are developed from bottom-up, from the small speech community to the more general ones. According to Graham (2007), emojis are 'glocalized.' Its use starts with the local community and 
adopted by more global communities. Hence, emojis are naturally used differently by people from various social group indicated by different ages, gender, geographical location, and status (Graham, 2019; Alshenqeeti, 2016). They show identity and membership to a particular speech community by serving as a phatic language (Graham, 2019). Interestingly, although emojis are usually developed by a local speech community, they also serve to address language barrier issues encountered by the digital community built by people from various socio-cultural backgrounds due to universality of visual, emotional messages (Graham, 2019). Furthermore, most emojis used today have been standardized by Google and Apple to ease universal understanding among people (Lucas, 2016).

Emojis are part of language that functions to deliver emotional situations and cues in CMC and are used differently by various groups of people (Alshenqeeti, 2016). In terms of frequency, for instance, people in Finland used emojis in their message the most frequently, while Tanzanians use emojis the least often compared to other 13 countries surveyed by Woollaston (2015). The types of emojis used by users in different countries are varied as well. French, for instance, uses more heartshaped emojis more frequently compared to other countries Woollaston (2015). The investigation on frequency and types of emojis used by people from various countries might indicate that certain symbols are more familiar to a particular culture. The more frequent use of heart-shaped emojis by French, for example, may confirm that Frenchs are romantic people. On the other hand, the least-frequent use of emojis by Tanzanian can also lead to research that aims to reveal the formality of CMC in Tanzania or Tanzanians' familiarity with pictorial languages.

In terms of perceived responsiveness, Coyle and Carmichael (2019) reported that emojis are only seen effective if they are used by both parties involved in the communication. When only one party uses emojis in the communication, the responsiveness is considered low compared to when neither party use emojis at all. Topic of the discussion also plays an important role in the perceived responsiveness of the use of emojis (Coyle and Carmichael, 2019). Discussions on positive issues tend to encourage more uses of emojis, while conversations on negative topics tend to discourage the use of emojis. When responding to adverse situations, people tend to show more serious tones that are not represented by emojis even when they portray sadness or grievance. However, the general notion of emojis as tools to enhance CMC might also be questioned as there is a condition where not using emojis at all can be more accommodating (Coyle \& Carmichael, 2019).

Related to the gender of the users, Alshenqeeti (2016) and Graham (2019) found that women tend to use more emojis than men. They subtly relate the results with the general belief that women tend to express their emotions more frequently and openly than men; thus, the emojis which one of the functions is to signify emotional messages are used more regularly. In terms of emojis used by other people to specific genders, such as the ones reported by Graham (2019) investigating how people use emojis when interacting with female and male live performing gamers, the findings demonstrate that when interacting with female gamers, the viewers tend to use emojis that indicate membership of particular gamers' community and playfulness. Whereas, when interacting with male live performing gamers, the viewers tend to use general emojis showing sarcasm and focus more on the performance.

When age variables are at play, Lu et al. (2016) reported that people aged 20-30 years old are the most frequent when it comes to using emojis in CMC. Prada et al. (2018) reported that young females use emojis more often than older females and males in general. In contrast, Nishimura (2015) reported that older females and males aged more 55 years and above tend to use more emojis than younger females and males.

The research reports above indicate that gender and age do not conclusively correlate with the types and functions of emojis used in communication. There are many possible intervening variables such as the exposure of speech community to technology, platforms and audiences that might influence the use of emojis. The research conducted by $\mathrm{Lu}$ et al. (2016, cited in Alshenqeeti, 2016), for instance, did not take into account the entire contexts of interaction. The data were collected from emoji entries in the Kika keyboard application in some countries. Hence, research focusing on age and gender might show different findings when the platforms and audiences are different.

This study aims to investigate the use of emojis by members of Indonesian Intergenerational WhatsApp group. WhatsApp has become one of the most popular platforms for communication in Indonesia since family usually makes a WhatsApp group as a platform to communicate or discuss family matters. The participants of the WhatsApp group vary in terms of age and gender, thus, interaction in the platform can generate data on the use of emojis by members from different age and gender group. Audiences in the WhatsApp group may have a different use of emojis as a form of communication style differs depending on various factors (Bell, 1984). Much of the research on the use of emojis by gender and age groups tend to use self-reporting or one on one interaction analysis (Riordan, 2017). Research on group interaction in WhatsApp is relatively scarce, especially in Indonesian context. Hence, this study investigates types and functions of emojis used by members of different ages and gender in one Indonesian family WhatsApp group. Studying the use of emojis in the Intergenerational WhatsApp group can shed light on how people from 
different ages and gender communicate in the digital forum. The findings can help CMC users to adapt their use of emojis according to age and genders of the forum member.

\section{METHOD}

The participants of this study are 42 members of an Indonesian Intergenerational WhatsApp group, as we can see in Table 1. In the coding process, initials are used to identify the participants. The age of the members varied. For convenience purposes, the participants aged 21-40 years old are categorized as younger participants, while the participants aged 41-70 are categorized as older participants. The languages used by the participants in WhatsApp interaction are Sundanese and Indonesian.

Firstly, the types of emojis are categorized based on Emojipedia (2019) accessed on https://emojipedia.org/whatsapp/. The emojis analyzed are those used in April-August 2019 group interaction. All emojis during that period are counted whether their occurrence is standalone or after written words. Then, the use of emojis will be categorized based on $\mathrm{Li}$ and Yang (2018) emoji function taxonomy, categorizing the function into 1) attitude/emotion signal, 2) attitude/emotion intensity enhancer, 3) illocutionary force modifier, 4) humor, 5) irony, 6) turn-taking/giving, and 7) backchanneling.

\section{FINDINGS AND DISCUSSIONS}

\subsection{Emoji Use in Indonesian Intergenerational WhatsApp Group}

The analysis of WhatsApp group conversation in April to August 2019 found that there are 501 emojis used by both men and women of all ages. In detail, men used 187 emojis, while women used 314 emojis during that period. The five most frequently used emojis of each gender are shown in the Table 1. Table 2 shows that both men and women in the WhatsApp group used the same types of emojis, namely 'folded hands' , beaming face with smiling eyes' $\theta$, 'palms up together, 'thumbs up' $\beta$, and 'face with hand over mouth'

The difference between emoji use by men and women is mainly in the frequency of certain emoji use. This tendency might indicate that the men and women in the WhatsApp group have different preferences of emotions signalled to the other members. However, as all participants are of Indonesian origin, a general understanding of the culture might be shared among members; hence, the same types of emoji used. Additionally, the findings also indicate the range of emojis used by each gender. The most used emoji by men is the 'folded hands' with 57 occurrences or $30.48 \%$ of the total.

Table 1. Participants of the research

\begin{tabular}{|c|c|c|c|c|c|c|c|c|c|c|c|}
\hline \multirow[b]{2}{*}{ Gender } & \multicolumn{10}{|c|}{ Age } & \multirow[t]{2}{*}{ Tota } \\
\hline & $21-25$ & $\begin{array}{l}26- \\
30\end{array}$ & $\begin{array}{l}31- \\
35\end{array}$ & $36-40$ & $\begin{array}{c}41- \\
45\end{array}$ & $\begin{array}{c}46- \\
50\end{array}$ & $\begin{array}{l}51- \\
55\end{array}$ & $\begin{array}{c}56- \\
60\end{array}$ & $\begin{array}{c}61- \\
65\end{array}$ & $\begin{array}{r}66- \\
70\end{array}$ & \\
\hline Men & 5 & 5 & 5 & 2 & & 2 & 3 & 2 & 2 & & 26 \\
\hline Women & & 2 & 4 & 1 & 2 & 1 & 3 & 1 & 1 & 1 & 16 \\
\hline Total & 5 & 7 & 9 & 3 & 2 & 3 & 6 & 3 & 3 & 1 & 42 \\
\hline
\end{tabular}

Table 2. The Five Most Used Emojis by Genders

\begin{tabular}{|c|c|c|c|c|c|c|}
\hline \multirow{2}{*}{ No. } & \multicolumn{3}{|c|}{ Men } & \multicolumn{3}{|c|}{ Women } \\
\hline & Emoji & Frequency & Percentage & Emoji & Frequency & Percentage \\
\hline 1 & 20 & 57 & $30.48 \%$ & & 46 & $14.65 \%$ \\
\hline 2 & & 36 & $19.25 \%$ & & 40 & $12.74 \%$ \\
\hline 3 & & 18 & $9.63 \%$ & & 34 & $10.83 \%$ \\
\hline 4 & & 16 & $8.56 \%$ & & 30 & $9.55 \%$ \\
\hline 5 & . & 15 & $8.02 \%$ & & 29 & $9.24 \%$ \\
\hline
\end{tabular}


Meanwhile, the most emoji used by women is 'the hand over mouth,' with 46 occurrences or $14.65 \%$ of the total emoji. The most frequently used emoji by women does not account for even $15 \%$ of the total emoji used. Furthermore, the data analysis demonstrates that the types of emojis used by women in this study are more varied. This tendency might show that women express more emotions or used various emojis to signal certain emotions in the WhatsApp group.

Lastly, less frequent used emojis such as 'baby' 'rose' , 'birthday cake' $\forall$, 'smiling face with sunglasses' note. The use of those emojis is much less frequent than other emojis, but they tend to indicate a special event that occurred in the conversation. The 'baby; emoji was used once when one of the members delivered a baby. The 'rose' and 'birthday cake' emojis were used four times when the participants congratulated members who had a birthday. The 'smiling face with sunglasses' emoji was used when talking about a vacation to a beach, while 'cold face' was used when talking about visiting a plateau. Those specific emojis may portray a cultural expression of the speech community. People from other cultures might not use the same emojis when expressing emotions in the same context. Cheng (2017) suggests that the Chinese use different emojis from European when congratulating others in $\mathrm{CMC}$.

\subsection{Gender, Age, and Use of Emojis}

In terms of age, this study found that men aged 21-25 years old used emojis the most frequently among men group. In the women group, women aged 51-55 years old used emojis the most often. The five most frequent emoji users by ages as we can see in Table 3 .

Table 3 indicates that the use of emoji is not directly correlated to age. This finding is in line with Alshenqeeti
(2016), stating that emoji is not a feature of CMC dominantly used by younger generations. They further explain that as long as users are exposed to technology, they can use emojis regardless of age.

There might also be other intervening variables at play. The power-relation (Holmes, 2001) among members and the idea of presence in the online chat group can also influence the frequency of emojis used by the members. The younger participants in the women groups might be reluctant to use emojis, or even to communicate in the group as the conversation mostly is dominated by the older generations. Most of the younger participants in the women groups are in-laws. On the other hand, most of the participants in men groups have direct family relations, such as being the sons, nephews, or grandsons. This family relationship and power-relation variables might generally affect the participants' contribution to the conversation. Less participation in the discussion then leads to fewer emojis found.

Other variables, such as how the participants treat any information in the online sphere, might also at play. According to Warren (2018) and Walther et al. (2018), people only respond to messages in online communication if they are prevalent and relevant to them. A family WhatsApp group will have members with a shared identity and family matters as the topic. However, in a Intergenerational family WhatsApp group, the issues can be quite broad. As plenty of issues in the WhatsApp group conversation in this study are related to distant family members related to older participants, the younger participants might not feel the urgency to involve in the discussion. They might also think that the older participants have represented them.

Lastly, variables related to the participants' socioeconomic status can also influence contribution in the group conversation as well as the use of emojis. In the men groups, the top users of emojis are those in the 2125 years old age group.

Table 3. The Five most Frequent Emoji Users by Ages

\begin{tabular}{ccccccc}
\hline & \multicolumn{2}{c}{ Men } & & \multicolumn{2}{c}{ Women } \\
\cline { 3 - 6 } No. & Ages & $\begin{array}{c}\text { Frequency of } \\
\text { emoji use }\end{array}$ & $\begin{array}{c}\text { Percentage of } \\
\text { emoji use }\end{array}$ & Ages & $\begin{array}{c}\text { Frequency } \\
\text { of emoji use }\end{array}$ & $\begin{array}{c}\text { Percentage } \\
\text { of emoji use }\end{array}$ \\
\hline 1 & $21-25$ & 79 & $42.25 \%$ & $51-55$ & 152 & $48.41 \%$ \\
2 & $26-30$ & 32 & $17.11 \%$ & $46-50$ & 68 & $21.66 \%$ \\
3 & $56-60$ & 31 & $16.58 \%$ & $56-60$ & 45 & $14.33 \%$ \\
5 & $31-35$ & 21 & $11.23 \%$ & $66-70$ & 36 & $11.46 \%$ \\
\hline
\end{tabular}


However, despite having five participants in the age group, only one participant was active and used emojis more frequently. In the women groups whose most frequent emoji users are from 51-55 years old age group, one participant contributed more than $90 \%$ of the emojis counted for those groups. Plenty of factors can affect the result, including the occupation of the participants. The most active participants in both men and women groups have the profession that allows them to contribute much to the conversation. They use more emojis because they have more time to be active in the conversation than other members.

\subsection{Functions of Emojis in the WhatsApp Group}

In terms of function, the emoji used in this study only served five functions out of seven functions of emoji in the taxonomy formulated by Li and Yang (2018). The functions of emojis in men groups are 1) attitude/emotion enhancer, 2) attitude/emotion signal, 3) backchanneling, 4) humor and 5) illocutionary force. The following are the samples of function found in the research.

\subsubsection{Attitudelemotion signal;}

(1) Nuju ameung ka mama Majalaya

'We are visiting grandma in Majalaya

The use of "face with hand over mouth" emoji is categorized as an attitude/emotion signal. Without it, other participants might now know if the sender is happy or not with the visit to grandma.

\subsubsection{Attitude/emotion intensity enhancer;}

$$
\text { 'Amin, makasih, SA }
$$

The 'folded hands' emoji, which can be interpreted as 'high five,' 'praying,' or 'thank you' is considered attitude/emotion intensity enhancer in the context above as the word 'Amen' and 'thank you' are sufficient to convey the message even without the emoji. The use of 'folded hands' emoji then is considered as an addition or enhancer.

\subsubsection{Illocutionary force modifier;}

(3) Waah gawat angpau* bisa2 hanguss... 'Damn, I might not get angpau ここ,

* envelope with money usually given during Muslim celebration by relatives with higher social and economic status.
In the expression above, the participant expressed her disappointment since she might not get the money due to several reasons. The frustration was genuine, as the angpau seems quite important for her. To soften the expression and to save both her faces and the one who should have given her the angpau, she used the 'pensive emoji.' The emoji is an illocutionary force used to ask the angpau giver to reconsider his decision indirectly.

\subsubsection{Humor;}

(4) Teu kaurus p sekda pensiun 1 bln ge... siga kolot pisan

'No one takes care of you anymore, Mr. government secretary, you retired only for one month, then you look much older now<smiles>c1ccc2ccccc2c1</smiles>

In the context above, the "face with hand over mouth" emoji shows emotion beyond the written utterances. As the feeling is humorous, the emoji is categorized as humor.

\subsubsection{Irony;}

BO mah angpauuu.saja.

'BO only thinks about angpauuu

The 'see-no-evil monkey' emoji, which usually is used to show embarrassment, above belongs to irony since the statement is clarified seriously by other members. It is not categorized as humor since the utterance and emoji are not contradictory. The emoji tends to emphasize that BO needs to feel ashamed of what she said; thus, an irony.

\subsubsection{Turn-taking/giving}

The turn-taking function was not found in the study. In general, the emoji functions as a turn-taking/giving signal when it is used as a response to the previous message before the user starts a new topic of conversation.

\subsubsection{Backchanneling;}

(4) ST: I heard that it would be in July

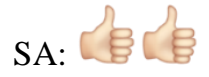

In the dialogue above, SA only responded by using 'thumbs up' emoji to signal that he has read the message from ST without further reply in written expression. Hence, it functions as a backchannel signal. 
Table 4. Function of Emojis Used by Men

\begin{tabular}{|c|c|c|c|c|c|c|c|c|c|c|c|c|}
\hline \multirow[b]{2}{*}{ No. } & \multirow[b]{2}{*}{ Functions } & \multicolumn{10}{|c|}{ Men } & \multirow[b]{2}{*}{ Total } \\
\hline & & $\begin{array}{l}21- \\
25\end{array}$ & $\begin{array}{l}26- \\
30\end{array}$ & $\begin{array}{l}31- \\
35\end{array}$ & $\begin{array}{l}36- \\
40\end{array}$ & $\begin{array}{l}41- \\
45\end{array}$ & $\begin{array}{l}46- \\
50\end{array}$ & $\begin{array}{l}51- \\
55\end{array}$ & $\begin{array}{l}56- \\
60\end{array}$ & $\begin{array}{l}61- \\
65\end{array}$ & $\begin{array}{l}\text { 66- } \\
70\end{array}$ & \\
\hline 1 & Attitude/emotion signal & 15 & 22 & 5 & 0 & 0 & 3 & 0 & 14 & 9 & 0 & 68 \\
\hline 2 & $\begin{array}{l}\text { Attitude/emotion } \\
\text { enhancer }\end{array}$ & 59 & 2 & 8 & 0 & 0 & 4 & 0 & 15 & 0 & 0 & 88 \\
\hline 3 & Illocutionary force & 0 & 2 & 0 & 0 & 0 & 0 & 0 & 0 & 0 & 0 & 2 \\
\hline 4 & Humor & 2 & 6 & 2 & 0 & 0 & 4 & 0 & 0 & 0 & 0 & 14 \\
\hline 5 & Irony & 0 & 0 & 0 & 0 & 0 & 0 & 0 & 0 & 0 & 0 & 0 \\
\hline 6 & Turn taking/giving & 0 & 0 & 0 & 0 & 0 & 0 & 0 & 0 & 0 & 0 & 0 \\
\hline \multirow[t]{2}{*}{7} & Backchannelling & 3 & 0 & 6 & 0 & 0 & 4 & 0 & 2 & 0 & 0 & 15 \\
\hline & Total & 79 & 32 & 21 & 0 & 0 & 15 & 0 & 31 & 9 & 0 & 187 \\
\hline
\end{tabular}

Table 5. Function of Emojis Used by Women

\begin{tabular}{|c|c|c|c|c|c|c|c|c|c|c|c|c|}
\hline \multirow[b]{2}{*}{ No. } & \multirow[b]{2}{*}{ Functions } & \multicolumn{7}{|c|}{ Women } & \multirow[b]{2}{*}{$\begin{array}{l}56- \\
60\end{array}$} & \multirow[b]{2}{*}{$\begin{array}{l}61- \\
65\end{array}$} & \multirow[b]{2}{*}{$\begin{array}{l}\text { 66- } \\
70\end{array}$} & \multirow[b]{2}{*}{ Total } \\
\hline & & $\begin{array}{l}21- \\
25\end{array}$ & $\begin{array}{l}26- \\
30\end{array}$ & $\begin{array}{l}31- \\
35\end{array}$ & $\begin{array}{l}36- \\
40\end{array}$ & $\begin{array}{l}41- \\
45\end{array}$ & $\begin{array}{l}46- \\
50\end{array}$ & $\begin{array}{l}51- \\
55\end{array}$ & & & & \\
\hline 1 & Attitude/emotion signal & 0 & 2 & 3 & 3 & 0 & 26 & 54 & 20 & 0 & 10 & 118 \\
\hline 2 & $\begin{array}{l}\text { Attitude/emotion } \\
\text { enhancer }\end{array}$ & 0 & 0 & 0 & 5 & 0 & 9 & 56 & 14 & 0 & 16 & 100 \\
\hline 3 & Illocutionary force & 0 & 0 & 0 & 0 & 0 & 0 & 4 & 2 & 0 & 0 & 6 \\
\hline 4 & Humor & 0 & 0 & 0 & 0 & 0 & 14 & 10 & 1 & 0 & 8 & 33 \\
\hline 5 & Irony & 0 & 0 & 0 & 0 & 0 & 0 & 0 & 0 & 0 & 2 & 2 \\
\hline 6 & Turn taking/giving & 0 & 0 & 0 & 0 & 0 & 0 & 0 & 0 & 0 & 0 & 0 \\
\hline \multirow[t]{2}{*}{7} & Backchannelling & 0 & 0 & 0 & 0 & 0 & 19 & 28 & 8 & 0 & 0 & 55 \\
\hline & Total & 0 & 2 & 3 & 8 & 0 & 68 & 152 & 45 & 0 & 36 & 314 \\
\hline
\end{tabular}

The frequency of the functions of emojis found in this research can be seen in Table 4 . This finding might indicate that men's emotional content in most messages could be understood more easily, even without the inclusion of emojis. This finding is in line with Tang and Hew (2019), stating that emoji has not been able to replace the role of words to convey messages. In the women group, there are six out of seven functions in the taxonomy (Li \& Yang, 2018) found in the study. Those functions are 1) attitude/emotion signal, 2) attitude/emotion enhancer, 3) backchanneling, 4) humor, 5) illocutionary force, and 6) irony. The details of the findings can be seen in Table 5.

Compared to the men, the women in this study tend to use more emojis to signal attitude or emotion. The finding might indicate that to convey the emotional load of the messages, adding emoji is necessary for women.
Furthermore, the use of irony in the women group can also be an interesting point to further research as it can indicate a particular communication style accommodated by emoji.

\section{CONCLUSION}

This study investigated the types and functions of emojis in the Indonesian Intergenerational WhatsApp group. This study found that the five most frequently used emojis by both men and women are 'folded hands', 'beaming face with smiling eyes', 'palms up together', 'thumbs up', and 'face with hand over mouth'. Furthermore, age might not correlate to the use of emojis when other factors such as power-relation, family relation, and occupation intervene. In terms of function, men tend to use more emojis to enhance attitude or 
emotion, while women tend to use more emojis to signal attitude or emotion.

As for the implication, this study shows that emojis are used and understood differently in different contexts as well as by different users. Understanding what emojis are frequently used in a particular community of speech can help users to navigate their communication strategy to create rapport or to avoid misunderstanding. From the study, for instance, CMC users might understand that to pray and apologize sincerely in the Intergenerational group should be signalled by the use of 'folded hands' emoji. CMC users can also see the importance of giving backchanneling in the form of emojis to make people feel heard and appreciated.

Nevertheless, the future further research needs to add more participants as having more participants and WhatsApp groups analysed will better reveal the pattern of the use of emojis. Comparing how the participants use emojis in different groups can also shed light on what variables encourage more use of emojis.

\section{REFERENCES}

Alshenqeeti, H. (2016). Are emojis creating a new or old visual language for new generations? A sociosemiotic study. Advances in Language and Literacy Studies, 7(6), 56-69.

Bell, A. (1984). Language style as audience design. Language in Society, 13(2), 145-204.

Cheng, D. L. (2017). Do I mean what I say and say what I mean? A cross-cultural approach to the use of emoticons and emojis in CMC messages. Fonseca, Journal of Communication, 15, 207-226.

Emojipedia (2019, September 6). Retrieved from https://emojipedia.org/whatsapp/2.19.244/

Coyle, M. A., \& Carmichael, C. I. (2019). Perceived responsiveness in text messaging: The role of emoji use. Computers in Human Behavior, 99, 181-189.

Graham, S. L. (2007). Disagreeing to agree: Conflict,(im) politeness and identity in a computer-mediated community. Journal of pragmatics, 39(4), 742-759.

Graham, S. L. (2019). A wink and a nod: The role of emojis in forming digital communities. Multilingua, 38(4), 377-400.

Gülşen, T. T. (2016). You tell me in emojis. In O. Takashi, \& A. Taisuke, Computational and Cognitive (pp. 356-378). New York: IGI Global.

Holmes, J. (2001). An introduction to sociolinguistics. Boston: Addison Wesley Publishing Company.

Skiba, D. J. (2016). Face with tears of joy is word of the year: are emoji a sign of things to come in health care?. Nursing education perspectives, 37(1), 5657.

Kavanagh, B. (2016). Emoticons as a medium for channeling politeness within American and Japanese online blogging communities. Language \& Communication, 48, 53-65.

Li, L., \& Yang, Y. (2018). Pragmatic functions of emoji in internet_based communication---a corpus- based study. Asian-Pacific Journal of Second and Foreign Language Education, 3(16), 1-12.

Lu, X., Ai, W., Liu, X., Li, Q., Wang, N., Huang, G., \& Mei, Q. (2016). Learning from the ubiquitous language: an empirical analysis of emoji usage of smartphone users. In Proceedings of the 2016 ACM International Joint Conference on Pervasive and Ubiquitous Computing, (pp. 770-780). Heidelberg.

Lucas, G. (2016). The story of emoji. London: Prestel Publishing.

McIntyre, E. S. (2016). From cave paintings to Shakespeare and back again: What are emoji and should I be afraid? (Doctoral dissertation). Texas State University, Texas. Retrieved from https://digital.library.txstate.edu/bitstream/handle/1 0877/6100/McIntyreEmily.pdf?sequence $=1$

Nishimura, Y. (2015). A sociolinguistic analysis of emoticon usage in Japanese blogs: Variation by age, gender, and. In Selected Papers of Internet Research 16: The 16th Annual Meeting of the Association of Internet Researchers.

Prada, M., Rodrigues, D. L., Garrido, M. V., Lopes, D., Cavalheiro, B., \& Gaspar, R. (2018). Motives, frequency and attitudes toward emoji and emoticon use. Telematics and Informatics, 35(7), 1925-1934.

Riordan, M. A. (2017). Emojis as tools for emotion work: Communicating affect in text messages. Journal of Language and Social Psychology, 36(5), 549-567.

Sampietro, A. (2016). Exploring the punctuating effect of emoji in Spanish Whatsapp chats. Lenguas Modernas, 47, 91-113.

Tang, Y., \& Hew, K. F. (2019). Emoticon, emoji, and sticker use in computer-mediated communication: A review of theories and research findings. International Journal of Communication, 13, 2457 2483.

Walther, J. B., Kashian, N., Jang, J.-W., Shin, S. Y., Dai, Y., \& Koutamanis, M. (2018). The effect of message persistence and disclosure on liking in computermediated communication. Media Psychology, 21(2), 308-327. 
Warren, A. N. (2018). Navigating assigned roles for asynchronous online discussions: Examining participants' orientation using conversation analysis. Online Learning Journal, 22(4), 27-45.

Woollaston, V. (2015). Are emoji killing off the alphabet? Instagram maps use of the icons to see how they're replacing internet slang words. Retrieved from http://www.dailymail.co.uk/sciencetech/article3070135/Are-emoji-killing-alphabet-Instagrammaps-use-iconsreplacing-internet-slang-words.html 